\title{
Atividades educativas em saúde bucal desenvolvidas por cirurgiões-dentistas com escolares: uma revisão sistematizada da literatura
}

\author{
Niliana Teixeira de Faria Campestrini*; Bárbara Munhoz da Cunha**; Prescila Mota de Oliveira \\ Kublitski***; Léo Kriger*****; Pablo Guilherme Caldarelli*****; Marilisa Carneiro Leão Gabardo******
}

* Cirurgiã-dentista, Unidade de Saúde Bom Pastor, Prefeitura Municipal de Curitiba

** Cirurgiã-dentista, Mestranda, Programa de Pós-Graduação em Odontologia, Universidade Positivo

*** Cirurgiã-dentista, Doutoranda, Programa de Pós-Graduação em Odontologia, Universidade Positivo

***** Cirurgião-dentista, Mestre, Professor aposentado, Pontifícia Universidade Católica do Paraná

***** Cirurgião-dentista, Pós-doutorando, Programa de PósGraduação em Odontologia, Universidade Positivo

****** Cirurgiã-dentista, Doutora, Professora, Programa de PósGraduação em Odontologia, Universidade Positivo

Recebido em 01/07/2019. Aprovado em 07/01/2020.

\begin{abstract}
RESUMO
Promover saúde bucal por meio da educação é uma estratégia possível de ser realizada por profissionais da área odontológica. No entanto, é tarefa desafiadora no que se refere à garantia de uma aprendizagem efetiva e transformadora de atitudes e de hábitos de vida. Esta revisão sistematizada teve como objetivo abordar o papel do cirurgião-dentista enquanto educador, capaz de atuar em ações voltadas para escolares, e em seguida fazer uma reflexão acerca do processo de formação profissional nesse contexto. Foram consultadas as bases LILACS e BBO via Biblioteca Virtual em Saúde e SciELO, com busca restrita para publicações entre os anos de 2009 a 2019, em língua portuguesa e realizadas no Brasil. Os descritores empregados foram educação, criança, saúde bucal e Odontologia. Foram selecionados e analisados nove artigos, os quais contemplavam atividades educativas em saúde bucal para crianças em escolas. Adicionalmente, foi feita uma reflexão sobre a capacitação do cirurgião-dentista, com base na nas Diretrizes Curriculares Nacionais em Odontologia. Observou-se que a ações educativas são de suma importância para as crianças, com impactos positivos em suas vidas, em condições clínicas e comportamentais. De modo reflexivo, coube um olhar crítico à fragilidade do que ainda é proposto durante a formação do cirurgião-dentista, mesmo mediante propostas curriculares atuais que almejam a expansão de competências e habilidades para fora do ambiente clínico. Concluiu-se que as pesquisas consultadas revelaram o aspecto positivo das ações em saúde bucal executadas. Contudo, sabe-se que, apesar do atual cenário estimulado por novas propostas curriculares, aos cirurgiões-dentistas ainda é custosa a transposição de barreiras que permitam maior engajamento em atividades educativas, que aqui foram reveladas como essenciais para o público escolar.

Descritores: Promoção da Saúde. Educação em Saúde. Escola. Pré-Escolar. Saúde Bucal. CirurgiãoDentista.
\end{abstract}




\section{INTRODUÇÃO}

Educação e saúde interagem nos espaços de produção de ações voltadas para a qualidade de vida da população. Contudo, o aprendizado sobre essa interação e a compreensão das consequências geradas por uma ou outra concepção pedagógica utilizada, predominantemente e ao longo do tempo, não têm o enfoque desejável na maior parte dos processos de formação dos profissionais da área da saúde ${ }^{1}$. Experiências mostram que transmitir informações a respeito do funcionamento da cavidade bucal e a descrição das características das doenças que a acometem, bem como um elenco de hábitos de higiene, não é suficiente para que as pessoas desenvolvam atitudes saudáveis ${ }^{2,3}$.

Para Brock et al. ${ }^{4}$ atuar na educação infantil é exercer um papel importante como mediador e facilitador da apropriação de conhecimentos mais elaborados pelas crianças, que aprendem de maneira lúdica de acordo com seu desenvolvimento físico, emocional, social e cognitivo. Por essa razão, educadores acreditam que a brincadeira é uma ferramenta valiosa para a aprendizagem por produzir uma motivação intrínseca. Para isso, ambientes divertidos que facilitem a promoção de atividades com uso de recursos interessantes são desejáveis 5 .

Estudos reconhecem que o ambiente escolar é privilegiado para práticas promotoras da saúde, como ações educativas e preventivas ${ }^{6-10}$. Vasconcelos et al. ${ }^{11}$ concluíram que a educação em saúde bucal deve estar presente na escola, por ter este espaço um papel na orientação e na formação cidadã das crianças.

Em 5 de dezembro de 2007 o Programa Saúde na Escola (PSE) foi instituído com a finalidade de contribuir para a formação integral dos estudantes da rede pública de educação básica, por meio de ações de prevenção, promoção e atenção à saúde ${ }^{12}$. As linhas de ação no que compete à saúde bucal dentro do PSE incluem a avaliação em saúde bucal, que é realizada uma vez ao ano de modo a identificar vulnerabilidades que necessitem de cuidados específicos. A partir daí, o planejamento das ações clínicas ocorre juntamente com a equipe de saúde. Por sua vez, as ações educativas e de promoção da saúde devem ser feitas em conjunto com a escola, sendo indispensável a presença do cirurgião-dentista e dos professores ${ }^{12}$. Quanto aos professores, há apontamentos na literatura que os mesmos precisam de educação continuada quanto à temática "saúde bucal" 13,14 .

As Diretrizes da Política Nacional da Saúde Bucal (PNSB) postulam que os conteúdos pedagógicos da educação em saúde bucal devem ser trabalhados de forma integrada com diversas áreas, nos formatos de debates, oficinas, teatros, dentre outros $^{15}$. Já as Diretrizes Curriculares Nacionais (DCN) para o ensino de graduação em Odontologia definem que a formação do cirurgião-dentista deve dotá-lo de competências e habilidades para a atenção à saúde tanto no nível individual quanto coletivo, para a tomada de decisões para a comunicação acessível e confiável, para a liderança com a equipe de trabalho, para administração e gerenciamento dos recursos humanos, físicos, materiais e de informação, e para a educação permanente ${ }^{16}$.

O profissional de saúde que deseja ter sucesso em sua intenção de ensinar deve estar estimulado e em sintonia com seu público-alvo para conseguir motivar e impulsionar mudanças. $\mathrm{O}$ interesse e a curiosidade despertados facilitam a aquisição de comportamentos positivos de saúde. Contudo, o cirurgião-dentista pode não se sentir preparado para a prática da educação infantil e manejo com crianças, além do desafio em ser criativo ao otimizar recursos restritos para atingir o objetivo de informar e o trabalho em equipe com profissionais de demais áreas.

Neste contexto, esta revisão sistematizada teve como objetivo avaliar atividades educativas em saúde bucal realizadas por cirurgiões-dentistas com escolares, bem como refletir como essas práticas são conduzidas no processo de formação profissional.

\section{METODOLOGIA}

A literatura foi revisada de modo sistematizado, por meio de consultas eletrônicas às 
bases de dados Literatura Latino-Americana e do Caribe em Ciências da Saúde (LILACS) e Bibliografia Brasileira de Odontologia (BBO), via Biblioteca Virtual em Saúde (BVS), e Scientific Electronic Library Online (SciELO). Foram feitas restrições (aplicação de filtros) de modo a recuperar apenas artigos publicados entre janeiro de 2009 e dezembro de 2019, em língua portuguesa e realizados no Brasil. Os descritores empregados foram educação, criança, saúde bucal e Odontologia, combinados com o uso do operador booleano "AND", para que todos estivessem presentes conjuntamente nos resultados. Foram selecionados e analisados artigos que contemplavam atividades educativas em saúde bucal para crianças em escolas, independentemente da idade das mesmas, do desenho de estudo adotado e de qual profissional realizou essas atividades, desde que a saúde bucal estivesse presente como temática. Apenas um autor fez a coleta, e a seleção e avaliação dos estudos foi pareada por dois autores familiarizados com o tema.

Os dados de autor/ano, amostra pesquisada, estratégia educativa empregada e resultados alcançados (por exemplo o impacto em condições clínicas, comportamentais, autopercepção, dentre outros) a que os autores chegaram, foram compilados. Dois autores realizaram essa etapa de modo independente, e um terceiro arbitrou o desempate nos casos em que alguma dúvida foi constatada, para que se chegasse a um consenso. Os itens foram agrupados e analisados de forma descritiva.

Em seguida, uma reflexão foi realizada acerca do processo de formação profissional, tendo como escopo as DCN para o curso de Odontologia.

\section{RESULTADOS}

Foram encontrados 63 artigos, sendo 57 na BVS e seis na SciELO. Nove exclusões foram feitas em decorrência de repetição de títulos. Pela leitura inicial dos títulos, três artigos foram descartados por serem revisões e 34 não tinham relação com o tema. Dezessete tiveram os resumos lidos e oito foram excluídos nessa fase por ainda não atenderem os critérios de inclusão. Ao final, nove artigos foram lidos na íntegra e incluídos na análise final (figura 1).

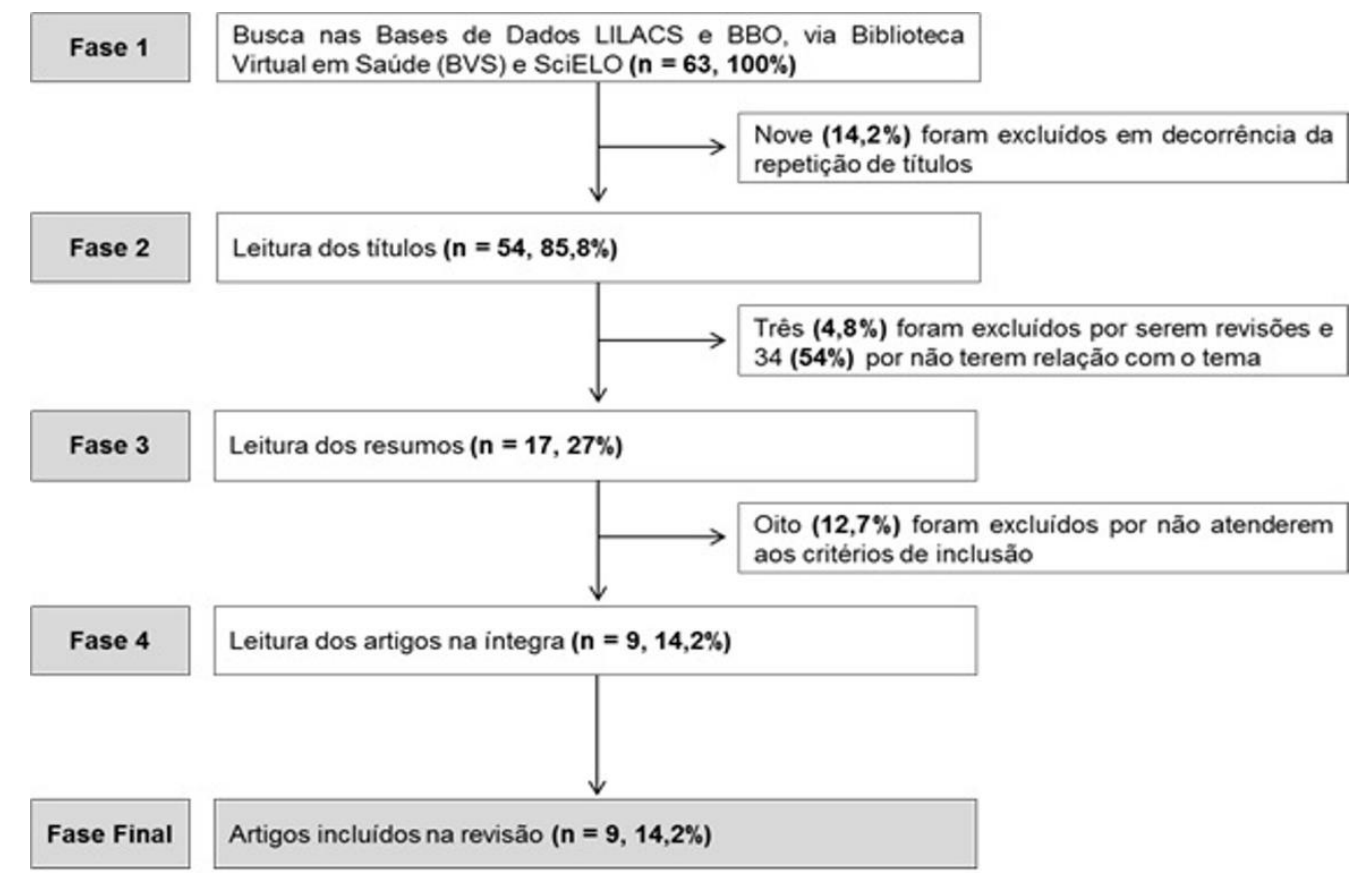

Figura 1. Busca, seleção, inclusão e exclusão dos estudos para a revisão sistematizada da literatura 
Os artigos selecionados e incluídos para análise encontram-se descritos na tabela 1 , a qual apresenta uma síntese dos principais aspectos desses estudos (ano, amostra, estratégia educativa utilizada e os resultados alcançados).

O tamanho das amostras variou entre 26 e 522 indivíduos.

Quanto às estratégias educativas, a orientação para higiene bucal (OHB) foi empregada em três estudos ${ }^{17-19}$, enquanto o uso de material educativo por meio de leitura foi utilizado em três estudos ${ }^{19-21}$. Já as atividades que promovem maior envolvimento, com participação ativa dos sujeitos, foram realizadas em cinco estudos ${ }^{17,20,22-}$ 24 .

Como resultados, todas as intervenções foram positivas, em termos de melhora de condição clínica ${ }^{17,19,20}$, aumento do nível de conhecimento a respeito do tema e mudança de comportamento $^{18,20-25}$.

Tabela 1. Síntese dos principais aspectos dos estudos incluídos na revisão sistematizada $(\mathrm{n}=9)$

\begin{tabular}{|c|c|c|c|}
\hline Autor/Ano & Amostra & Estratégia educativa & Resultados alcançados \\
\hline Borges et al. $/ 2009^{17}$ & 120 & Palestra, teatro e OHB. & $\begin{array}{l}\text { Redução de biofilme, } \\
\text { problemas gengivais e cárie. }\end{array}$ \\
\hline Garcia et al. $/ 2009^{20}$ & 120 & $\begin{array}{l}\text { Leitura material auto instrutivo } \\
\text { (Gibi), discussão em grupo e } \\
\text { atividade de fixação de conteúdo. }\end{array}$ & $\begin{array}{l}\text { Redução de biofilme, em } \\
\text { especial nos grupos em que } \\
\text { as estratégias foram unidas. }\end{array}$ \\
\hline Orsi et al. $/ 2009^{25}$ & 386 & $\begin{array}{l}\text { Palestras com uso de material } \\
\text { lúdico. }\end{array}$ & $\begin{array}{l}\text { Maior adesão a hábitos de } \\
\text { higiene bucal. }\end{array}$ \\
\hline Ribeiro et al. $/ 2009^{22}$ & 33 & $\begin{array}{l}\text { Método educativo em sala de aula } \\
\text { por meio de seminários } \\
\text { apresentados pelos alunos. }\end{array}$ & $\begin{array}{l}\text { Aumento do conhecimento } \\
\text { em todos os temas abordados } \\
\text { após a aplicação do método. }\end{array}$ \\
\hline Valarelli et al./2011 $1^{18}$ & 522 & OHB e uso de material lúdico. & $\begin{array}{l}\text { Maior adesão a hábitos de } \\
\text { higiene bucal e redução de } \\
\text { doenças. }\end{array}$ \\
\hline Barreto et al. $/ 2013^{19}$ & 38 & $\begin{array}{l}\text { OHB, recebimento de kits de } \\
\text { higiene e material educativo. }\end{array}$ & $\begin{array}{l}\text { Diminuição significativa de } \\
\text { biofilme no grupo que } \\
\text { participou das atividades. }\end{array}$ \\
\hline Carvalho et al. $/ 2013^{23}$ & 169 & Atividades lúdicas. & $\begin{array}{l}\text { Maior adesão a hábitos de } \\
\text { higiene bucal. }\end{array}$ \\
\hline Castro e Costa/2015 21 & 26 & $\begin{array}{l}\text { Material instrutivo por meio de } \\
\text { Literatura de Cordel. }\end{array}$ & $\begin{array}{l}\text { Estratégia pedagógica } \\
\text { criativa e dinâmica que } \\
\text { auxiliou na motivação, } \\
\text { educação e promoção da } \\
\text { saúde bucal. }\end{array}$ \\
\hline Figueiredo et al. $/ 2015^{24}$ & 45 & $\begin{array}{l}\text { Utilização de um software } \\
\text { educativo com temática relacionada } \\
\text { à higiene bucal. }\end{array}$ & $\begin{array}{l}\text { A ferramenta de ensino foi } \\
\text { importante para motivar os } \\
\text { cuidados em saúde bucal. }\end{array}$ \\
\hline
\end{tabular}

OHB: Orientação para Higiene Bucal

\section{DISCUSSÃO}

Aprender a ensinar, elaborar atividades condizentes com a fase de desenvolvimento da criança e expandir a sua criatividade são, com certeza, desafios presentes na rotina do profissional que pratica ações educativas em ambiente escolar. 
Práticas de educação em saúde bucal visam levar a informação adequada ao indivíduo, motivando-o e auxiliando no desenvolvimento de habilidades para o autocuidado e incorporação de hábitos saudáveis ${ }^{7}$. Pesquisas da década de 1990 apontam que há maior probabilidade de manutenção de estabilidade da saúde quanto mais precocemente os comportamentos saudáveis forem inseridos na infância ${ }^{5}$.

Estudos mostram que a educação em saúde bucal nas escolas reduz significativamente $o$ surgimento de agravos bucais, a exemplo da cárie dentária, o que sugere uma integração destas ações ao currículo, impactando positivamente no conhecimento, atitudes e práticas de prevenção ${ }^{7,17}$. Desta forma, o ambiente escolar é propício para as atividades de promoção da saúde, com abordagens educativas e preventivas ${ }^{7-10,18}$. Uma recente revisão sistemática com metanálise a respeito da efetividade da educação em saúde bucal em escolares indicou que as ações "tradicionais", ou seja, aquelas com base na transmissão de conhecimentos, são capazes de melhorar o controle de placa, mas não de gengivite e de cárie dentária. Os autores ainda afirmam que, em longo prazo, não existem evidências concretas que as intervenções no ambiente escolar tenham resultados positivos ${ }^{26}$.

Dentre as práticas pedagógicas com o tema "Saúde Bucal", há uma gama de recursos que podem ser realizados, tais como teatros e oficinas, dentre outros, inclusive sendo estimulados pela $\mathrm{PNSB}^{15}$. Pelos estudos avaliados, o uso de recursos lúdicos foi relevante, o que sugere sua importância para essa população ${ }^{17,18,20,21,23-25}$. Pinto e Tavares $^{27}$ esclarecem que o lúdico melhora resultados de aprendizagem, enquanto proporciona a interação da criança com o ambiente. Para as autoras, as atividades lúdicas permitem a incorporação de valores, a assimilação de novos conhecimentos o desenvolvimento cultural, social e da criatividade, de maneira prazerosa.

Valarelli et $a .^{18}{ }^{18}$ em estudo sobre importância dos programas de educação e motivação para saúde bucal em escolas, reforçam que a educação e a motivação estabelecida nessa fase impactam sobre o desempenho da criança e, que para que haja sucesso deste trabalho, as inovações nas atividades dependem da habilidade do profissional. Outros autores corroboram esses achados, visto que as atividades desenvolvidas motivaram os escolares e auxiliaram nas mudanças de hábitos ${ }^{18,21,23-25}$.

Em pesquisa de Promoção de Saúde Bucal, com a finalidade de captar informações sobre as atividades lúdicas e materiais educativos prediletos de crianças entre quatro e seis anos, autores coletaram dados com educadoras em uma creche de Piracicaba, São Paulo, e obtiveram como resultado que o teatro com fantoches ou com atores, as atividades com música e a exibição de desenhos animados foram as estratégias lúdicas preferidas pelas crianças 5 .

Para Morano Júnior e Mialhe ${ }^{28}$ o uso da música também é um recurso interessante, por meio do qual conceitos em saúde bucal podem ser aprendidos. Já Antonio et $a l^{6}{ }^{6}$, na avaliação de diferentes métodos educativos em saúde bucal com crianças de sete a dez anos de idade em uma escola pública na cidade de Araras, São Paulo, concluíram que dentre os métodos aplicados de teatro, gincana, palestra e história em quadrinhos, este último foi o que teve mais resultados positivos. Os autores complementaram que a seleção dos métodos a serem utilizados está na dependência direta da faixa etária, da condição socioeconômica, do local e do assunto a ser abordado, e que se deve sempre usar recursos audiovisuais com personagens infantis atuais. Nessa mesma linha, Malik et al. ${ }^{29}$ perceberam que jogos interativos e palavras cruzadas com conteúdo relacionado à saúde bucal foram uma forma fácil e efetiva na construção do conhecimento com crianças entre oito e 12 anos de idade, em comparação à forma tradicional de ensinar, com apresentações orais.

Destaca-se, ainda, um projeto de extensão desenvolvido por estudantes de graduação e de pós-graduação, voltado para e educação em saúde bucal em escolares, de uma universidade de Santa 
Catarina. Os autores concluíram que tal ação, com oficinas com material lúdico e criativo, colaborou para a formação profissional dos envolvidos, superando os limites puramente técnicos e clínicos ${ }^{8}$.

Além de integrar e articular as políticas de saúde e educação, o PSE surge como uma estratégia que aproxima as equipes das unidades de saúde da família com o corpo docente da escola, seus estudantes e familiares, estreitando laços necessários para efetividade das ações em saúde. Unir as ações do Sistema Único de Saúde (SUS) com as ações das redes de educação básica pública é uma forma de ampliar o alcance e o impacto sobre as vulnerabilidades em saúde que comprometem o desenvolvimento escolar ${ }^{12}$.

Cabe às secretarias Estaduais e Municipais de Educação e Saúde, juntamente com as escolas, definir as prioridades e metas de atendimento do programa. As ações, inclusive com enfoque bucal, previstas no PSE, consideram aspectos de atenção, prevenção, promoção e manutenção da saúde no âmbito escolar, com a inclusão dessa temática no projeto político pedagógico das escolas ${ }^{12}$.

Embora ainda com muitas fragilidades e limitações, o PSE se apresenta como uma realidade nacional e em movimento de constante ampliação e transformação ${ }^{30}$. Com o intuito de tentar superar tais limitações, Milanez et al. ${ }^{10}$, desenvolveram uma plataforma educacional virtual (SB na escola), utilizando diferentes tecnologias de informação e comunicação, para difundir conhecimento relacionado à Odontologia na comunidade, entre gestores e profissionais da saúde e educação atuantes no PSE. Essa ferramenta pareceu facilitar o acesso à informação e colaborar para a promoção da saúde e educação da população.

Com referência à formação em Odontologia e o trabalho em ações educativas coletivas, o ensino superior nessa área vem sendo objeto de debate em relação ao modelo tradicional de ensino e de formação profissional. Reflexões acerca de novas metodologias de ensino, que possibilitem ao estudante desenvolver competências generalistas, interdisciplinares, criativas e resilientes, de forma a se tornarem capazes de atuar em diferentes equipamentos sociais, vem ganhando destaque ${ }^{31}$.

Farias e Sampaio $^{32}$ concluíram que a formação profissional, baseada na visão da doença como fenômeno estritamente biológico, influencia no processo de trabalho e impede a troca de saberes com outros profissionais. Essa visão reducionista tenta ser ultrapassada com o disposto nas DCN dos cursos de graduação em Odontologia, onde se espera que o egresso tenha competência e habilidades voltadas para atenção à saúde e comunicação. Um exemplo prático dessa discussão foi o trabalho de Cawahisa et $a l^{33}$, onde acadêmicos do $3 .^{\circ}$ e $4 .^{\circ}$ anos do curso de Odontologia desenvolveram atividades de promoção e prevenção em saúde bucal em uma escola de educação infantil. A interação entre atividades educativas e curativas, segundo os autores, possibilitou o desenvolvimento do proposto nas DCN, o que favorece a formação de um profissional diferenciado.

É urgente que o conceito de clínica ampliada seja incorporado, para que os estudantes de Odontologia possam visualizar seu campo de atuação de forma diversificada ${ }^{34}$.

\section{CONCLUSÕES}

As ações educativas em saúde bucal desenvolvidas com escolares promoveram conquista de hábitos saudáveis, inclusive com melhora de aspectos clínicos. O cirurgião-dentista deve estar ciente do seu papel como educador, capaz de instrumentalizar e assim empoderar os indivíduos quanto ao processo saúde-doença. Nesse contexto, as DCN trazem à tona a necessidade de um profissional com visão ampliada sobre suas habilidades e competências, que incluem o engajamento nesse tipo de ação.

\section{ABSTRACT \\ Oral health educational activities developed by dentists with students: a systematized literature review}


Dentists can effectively promote oral health through education. However, this is challenging in terms of ensuring effective and transformative learning attitudes and lifestyle habits. This systematized review addressed the role of the dentist as an educator capable of promoting student action while further reflecting on the professional training process in the same context. The LILACS and BBO databases were thus consulted through the Biblioteca Virtual em Saúde and SciELO. Searches were restricted to publications from 2009 to 2019 that were written in Portuguese and conducted in Brazil. The following search terms were used: education, childhood, oral health, and dentistry. A total of nine returned articles dealing with educational activities designed to teach oral health to schoolchildren were selected for analysis. A subsequent reflection was conducted to assess dentist qualifications according the National Curricular Guidelines on Dentistry. It was observed that educational actions were extremely important for children, with positive impacts on their lifestyles in regard to both clinical and behavioral conditions. A careful assessment was conducted regarding the elements proposed to dentists during training, including current curricular proposals aimed at expanding competencies and skills outside the clinical setting. There were positive aspects related to oral health actions in this regard. However, dentists are still expected to transpose barriers that allow greater participation in educational activities despite the current environment created by new curricular proposals, which this study found were essential for schoolchildren.

Descriptors Health Promotion. Health Education. School. Preschool Child. Oral Health. Dentist.

\section{REFERÊNCIAS}

1. Vasconcelos M, Grillo MJC, Soares SM. Práticas pedagógicas em Atenção Básica à Saúde. Tecnologias para abordagem ao indivíduo, família e comunidade. Unidade Didática I - Organização do processo de trabalho na Atenção Básica à Saúde. Belo Horizonte: Editora UFMG/Nescon - UFMG; 2009. 72 p.
2. Alves MU, Volschan BCG, Haas NAT. Educação em saúde bucal: sensibilização dos pais de crianças atendidas na clínica integrada de duas universidades privadas. Pesq Bras Odontoped Clin Integr. 2004; 4(1): 47-51.

3. Pauleto ARC, Pereira MLT, Cyrino EG. Saúde bucal: uma revisão crítica sobre programações educativas para escolares. Ciênc saúde coletiva. 2004; 9(1): 121-30.

4. Brock A, Dodds S, Jarvis P, Olusoga Y. Brincar - Atividade para a vida. Porto Alegre: Artmed; 2011. 432 p.

5. Venâncio DR, Gibilini C, Batista MJ, Gonçalo CS, Sousa MLR. Promoção da saúde bucal: desenvolvendo material lúdico para crianças na faixa etária pré-escolar. J Health Sci Inst. 2011; 29(3): 153-6.

6. Antonio LP, Gouvêa GR, Souza LZ, Cortellazzi KL. Avaliação de diferentes métodos educativos em saúde bucal em crianças na faixa etária de 7 a 10 anos de idade. RFO UPF. 2015; 18(2): 52-8.

7. Haque SE, Rahman M, Itsuko K, MutaharaM, Kayako S, Tsutsumi A, et al. Effect of a school-based oral health education in preventing untreated dental caries and increasing knowledge, attitude, and practices among adolescents in Bangladesh. BMC Oral Health. 2016; 16:4 4.

8. Carcereri DL, Peres ACO, Ludwig CP, Oliveira TFS, Menosso AG, Bortoli JQ, et al. Práticas inovadoras de educação em saúde bucal para promoção da saúde: relato de experiência. Extensio: R Eletr de Extensão. 2017; 14(26): 143-51.

9. Bausen AG, Scarparo A, Barcelos R. A eficiência da promoção da saúde bucal no ambiente escolar: revisão de literatura. Rev Bras Odontol. 2018; 75 (Supl. 2): 53.

10. Milanez LMPF, Berry M; Souza MIC, Cardoso AL. SB na escola: development of an educational platform for users of the 'Programa Saúde na Escola'. Rev Bras Odontol. 2018; 75: e1176. 
11. Vasconcelos R, Matta ML, Pordeus IA, Paiva SM. Escola: um espaço importante de informação em saúde bucal para a população infantil. PGR-Pós-Grad Rev Fac Odontol São José dos Campos. 2001; 4(3): 43-51.

12. Brasil. Presidência da República. Casa Civil. Subchefias de assuntos jurídicos. Decreto $\mathrm{n}^{\circ}$ 6.286 de 5 de dezembro de 2007. Institui o Programa Saúde na Escola e dá outras providências. Diário Oficial da União. Brasília 6 de dezembro de 2007. Seção1. p. 2.

13. Costa MM, Barbosa ADL, Fernandes JMFA, Fonseca FRA, Paredes SO. Conhecimento e práticas em saúde bucal nas escolas de ensino fundamental de um município de pequeno porte do sertão paraibano. Arq Odontol. 2014; 50(04): 193-202.

14. Oliveira EL, Riatto SG, Vieira APSB, Carvalho G, Fonseca M, Guedes V, et al. A importância do nível de conhecimento dos professores de escola pública do ensino fundamental sobre saúde bucal - revisão de literatura. Revista Campo do Saber. 2018; 4(5): 2-16.

15. Brasil. Ministério da Saúde. Secretaria de Atenção à Saúde. Departamento de Atenção Básica. Coordenação Nacional de Saúde Bucal. Diretrizes da Política Nacional de Saúde Bucal. Brasília; 2004.

16. Brasil. Ministério da Educação. Conselho Nacional da Educação. Resolução CNE/CES3 de 19 de fevereiro de 2002. Institui Diretrizes Curriculares Nacionais do Curso de Graduação em Odontologia. Diário Oficial da União. Brasília, 4 de março de 2002. seção 1, p.10.

17. Borges BCD, Trindade FCS, Silva RSG, Fernandes MJM, Costa ICC, Pinheiro IVA. A escola como espaço promotor de saúde bucal: cuidando de escolares por meio de ações coletivas. Rev Baiana Saúde Pública. 2009; 33(4)? 642-53.

18. Valarelli FP, Franco RM, Sampaio CC, Mauad C, Passos VAB, Vitor LLR, et.al.
Importância dos programas de educação e motivação para saúde bucal em escolas: relato de experiência. Odontol Clín-Cient. 2011; 10(2): 173-6.

19. Barreto DM, Paiva SM, Ramos-Jorge ML, Ferreira MC. Avaliação da eficácia de uma atividade educativo-preventiva com préescolares: estudo piloto de um ensaio clínico randomizado. Arq Odontol. 2013; 49(3): 11321.

20. Garcia PPNS, Nogueira I, Dovigo LN, Dotta EAV, Dovigo MRPN, Nassour EISC, et al. Educação em saúde: efeito de um método de auto-instrução sobre os níveis de higiene oral em escolares. Pesqui Bras Odontopediatria Clín Integr. 2009; 9(3): 333-7.

21. Castro MCS, Costa ICC. A literatura de cordel como instrumento didático-pedagógico na educação, motivação e promoção da saúde bucal. Rev Ciênc Plur. 2015; 1(1): 40-9.

22. Ribeiro DG, Dovigo LN, Silva SRC. Avaliação de um método educativo em saúde bucal aplicado em escolares de ensino público. Arq Odontol. 2009; 45(3): 154-9.

23. Carvalho THL, Pinheiro NMS, Santos JMA, Costa LED, Queiroz FS, Nóbrega CBC. Estratégias de promoção de saúde para crianças em idade pré-escolar do município de Patos-PB. Rev Odontol UNESP. 2013; 42(6): 426-31.

24. Figueiredo MC, Garcia M, Barone DAC, Oliveira R, Lurmmerz G. Gamificação em saúde bucal: experiência com escolares de zona rural. Rev ABENO. 2015; 15(3): 98-108.

25. Orsi VME, Pereira AA, Flório FM, Souza LZ, Boaretto P, Pinheiro PPS, et al. Hábitos e conhecimentos de escolares sobre saúde bucal. RGO - Rev Gaúcha Odontol. 2009; 57(3): 291-6.

26. Stein C, Santos NML, Hilgert JB, Hugo FN. Effectiveness of oral health education on oral hygiene and dental caries in schoolchildren: Systematic review and meta-analysis. Community Dent Oral Epidemiol. 2018; 
46(1): 30-7.

27. Pinto CL, Tavares HM. O lúdico na aprendizagem: apreender e aprender. Revista da Católica, Uberlândia. 2010; 2(3): 226-35.

28. Morano Junior M, Mialhe FL. A importância da professora na promoção de saúde bucal dos escolares. Rev Odontol Univ Cid São Paulo. 2008; 20(1): 19-22.

29. Malik A, Sabharwal S, Kumar A, Singh Samant P, Singh A, Kumar Pandey V. Implementation of game-based oral health education vs conventional oral health education on children's oral health-related knowledge and oral hygiene status. Int J Clin Pediatr Dent. 2017; 10(3): 257-60.

30. Vieira LS, Saporetti GM, Belisário SA. Programa saúde na escola: marcos jurídicos e institucionais. Rev Med Minas Gerais. 2016; 26 (Supl 8): S381-7.

31. Mello CCB, Alves RO, Lemos SMA. Metodologias de ensino e formação na área da saúde: revisão de literatura. Rev CEFAC. 2014; 16(6): 2015-28.
32. Farias MR, Sampaio JJC. Papel do cirurgiãodentista na equipe de saúde da família. Rev Gaúcha Odontol. 2011; 59(1): 109-15.

33. Cawahisa PT, Terada RSS, Pascotto RC, Occhi IG, Fujimaki M. Atividades realizadas durante o estágio supervisionado do curso de odontologia da UEM em um centro de educação infantil. Ciênc Cuid Saúde. 2013; 12(2): 375-81.

34. Brasil. Ministério da Saúde. Secretaria de Atenção à Saúde. Departamento de Atenção Básica. A saúde bucal no Sistema Único de Saúde / Ministério da Saúde, Secretaria de Atenção à Saúde, Departamento de Atenção Básica. - Brasília: Ministério da Saúde; 2018.

\section{Correspondência para:}

Marilisa Carneiro Leão Gabardo

e-mail: marilisagabardo@gmail.com

Rua Prof. Pedro Viriato Parigot de Souza, 5300

81280-330 Curitiba/PR 